\title{
SSRAT: The processing of rating scales for the determination of two-dimensional sociometric status
}

\author{
GERARD H. MAASSEN and JOHANNES A. LANDSHEER \\ Utrecht University, Utrecht, The Netherlands
}

\begin{abstract}
SSRAT is an MS-DOS computer program for two-dimensional sociometric status determination with rating scales. It classifies members of a population into five sociometric status groups, using 3-, 5-, 7-, or 9-point ratings in matrices of assessors and assessed. The processing of rating scales instead of nominations offers several advantages, including increased variability and reliability of the resulting scores. SSRAT is built on the null hypothesis of conditional random attribution of ratings, according to the particular probability distribution of an assessor. Output is available in the form of an ASCII SPSS data file for further statistical processing. Recent studies show that SSRAT, in comparison with nomination methods, (1) is superior in determining popular persons while it detects rejected persons as well, and (2) probably yields classifications that have a higher temporal stability.
\end{abstract}

Sophisticated techniques developed over the past two decades, such as multidimensional scaling by means of correspondence analysis, have been applied successfully to the analysis and spatial visualization of the structures of social groups. Yet there remains a need for diagnostic methods that yield results both easily interpretable and readily applicable in further research. For example, in the fields of developmental psychology and education, considerable research has been devoted to youths who have problems in relations with their peers and to the possibilities of adapting their social behavior. The sociometric methods currently in use to identify such youths (Coie, Dodge, \& Coppotelli, 1982; Newcomb \& Bukowski, 1983) are based on nominations: respondents are asked to nominate a prescribed number of group mates whom they like least and whom they like most. The "least liked" and "most liked" nomination totals received by a person classify him/her according to one of the following five categories: popular, rejected, average, neglected, or controversial. Typically, researchers pay special attention to those who are rejected or neglected; the popular are often included in the study as a reference group.

There are several drawbacks to such nomination procedures (Maassen, van der Linden, \& Akkermans, 1997). First, there is a conceptual problem. When an assessor is asked to nominate a prescribed number (typically three) of "least liked" and "most liked" group members, he/she is forced to split his/her individual latent continuum of peers liked and disliked into three parts, which for convenience can be indicated as $-1,0,+1$. In the

Correspondence should be addressed to G. H. Maassen, Department of Methodology and Statistics, Faculty of Social Sciences, Utrecht University, P.O. Box 80140, 3508 TC Utrecht. The Netherlands (e-mail: g.maassen (afss.uu.nl). nomination procedure, each assessor is asked to grant the prescribed number of individuals the score -1 and the same number of individuals the score +1 . For example, if the judge dislikes nobody, all latent scores lie on the positive side of the continuum. In this case, a score of -1 has to be granted to an individual who is in fact more or less liked, even though the score represents a (negative) preference. In effect, the nominations express the feelings of the assessor in a distorted way. In our view, if a unidimensional model of attributing liking scores to group peers is assumed on the level of the individual assessor, the obvious thing to do is to instruct respondents accordingly. Rating scales are more suitable for this than nominations.

A second major drawback of the nomination procedure is that only limited information is collected and processed. Nomination scores are dichotomous (nominated/ not nominated); ordinal information is lacking. It may be that sympathy differs for nominated persons, or for nonnominated persons, but this cannot be made explicit. With rating scales, on the other hand, respondents can give a more refined judgment about their group peers.

In comparison with that of nominations, the test-retest reliability of peer ratings is superior (Asher \& Hymel, 1981). The score that is traditionally derived from rating scales, the average received rating (arr), is highly stable over time even with young children (Asher, Singleton, Tinsley, \& Hymel, 1979). Nevertheless, in a study in which the temporal stability of the ratings- and nominationbased measures for sociometric status were compared for the first time, Terry and Coie (1991) found no appreciable difference. They do not show much enthusiasm for the ratings procedure because it entails a one-dimensional classification into sociometric status groups. Terry and Coie "conclude that groups formed on the basis of a onedimensional variable will have less desirable psychome- 
tric properties than those formed by a two-dimensional system" (p. 879).

In several kinds of investigations, however, peer status operationalized as a continuous variable is the appropriate measure for further analysis (such as correlational and regression analyses or comparisons over groups and over time). In particular, longitudinal designs are becoming increasingly popular among child and youth researchers. Because the nomination methods allow each assessor to attribute only a few nominations to peers, nomination totals are generally low and less discriminating than rating totals. If research groups are relatively stable and the children studied know each other well, ratings might be the method of choice, because each rating would add information (Terry \& Coie, 1991).

Yet although rating scales should be given preference in many situations, researchers may be inclined to consider the one-dimensionality of such classification a decisive drawback. In this article, a method and computer program (SSRAT) are presented that enable the researcher to process rating scores in order to yield the familiar twodimensional classification into five sociometric status groups. A recent study (Maassen, Goossens, \& Bokhorst, 1998 ) in which two-dimensional classifications based on nominations and on SSRAT are compared indicates a higher temporal stability for the ratings procedure.

\section{Determination of Sociometric Status}

Since the beginning of the 1980 s, two nomination procedures for the determination of sociometric status have predominated. The first phase in the application of both methods is that respondents belonging to a social group (often a classroom group) are asked two questions: (1) Which group members do you like most? And (2) which group members do you dislike most? Let LM be the number of "most liked" nominations received by a person and LL the number of "least liked" nominations received. In the standard score method, presented by Coie et al. (1982; slightly modified by Coie \& Dodge, 1983), LM and LL are standardized within the group. Next, the scores for social preference and social impact are calculated as the difference and sum, respectively, of these standard scores (i.e., $\mathrm{SP}=z_{\mathrm{LM}}-z_{\mathrm{LL}}$ and $\mathrm{SI}=z_{\mathrm{LM}}+z_{\mathrm{LL}}$ ) and are standardized as well. The attribution of sociometric status in terms of the model is as follows:

$$
\begin{array}{lll}
\text { 1. popular: } & z_{\mathrm{SP}}>1, \quad z_{\mathrm{LM}}>0 \text { and } z_{\mathrm{LL}}<0 ; \\
\text { 2. rejected: } & z_{\mathrm{SP}}<-1, \quad z_{\mathrm{LM}}<0 \text { and } z_{\mathrm{LL}}>0 ; \\
\text { 3. neglected: } & z_{\mathrm{SI}}<-1, \quad z_{\mathrm{LM}}<0 \text { and } z_{\mathrm{LL}}<0 ; \\
\text { 4. controversial: } & z_{\mathrm{SI}}>1, \quad z_{\mathrm{LM}}>0 \text { and } z_{\mathrm{LL}}>0 ; \\
\text { 5. average: } & \text { remaining group members. }
\end{array}
$$

In contrast, Newcomb and Bukowski (1983) proposed a probability method, in which nonstandardized scores are processed. In addition to the scores LM and LL only the impact score $\mathrm{SI}=\mathrm{LM}+\mathrm{LL}$ is used. Given the instruction to the respondents to nominate a prescribed number $a$ of "least liked" and "most liked" nominations, under the null hypothesis that nominations are randomly assigned, the probability that a group member receives a "most liked" nomination from a fellow group mate is fixed: $a /(N-1)$, where $N$ is the group size. The same holds for the probability of receiving a "least liked" nomination. Thus, under the null hypothesis, for each group member, LM and LL are the sums of $N-1$ Bernoulli variables. If the assumption is made that one will not give a fellow member both a "most liked" nomination and a "least liked" nomination, the impact score SI may be viewed as the sum of $N-1$ Bernoulli variables too. In the model of Newcomb and Bukowski, the probabilities of realized LM, $\mathrm{LL}$, and SI scores are compared with a chosen significance level. In this model, the sociometric status is attributed as follows:

$$
\begin{array}{ll}
\text { 1. popular: } & \text { LM significantly high, LL }<\mathscr{E L L} \text {; } \\
\text { 2. rejected: } & \text { LL significantly high, LM }<\mathscr{E} \mathrm{LM} \text {; } \\
\text { 3. neglected: } & \text { SI significantly low; } \\
\text { 4. controversial: } & \text { LM significantly high, LL }>\mathscr{E} \mathrm{LL},
\end{array}
$$

or

$$
\text { LL significantly high, LM }>\mathscr{E} \text { LM; }
$$

\section{5. average: remaining group members.}

In recent years, studies in which sociometric status has been determined by means of the nomination methods have proliferated in the field of child and youth research (Newcomb, Bukowski, \& Pattee, 1993).

\section{Theoretical Background}

The rating-scale method that we propose, SSRAT, is based on the same principle as is the nomination procedure, and it contains elements of both methods. Suppose that each group member is asked to rate all of his/her fellow group members on a scale ranging from disliking very much through a neutral scale midpoint to liking very much. In general terms, we assume that the data are gathered on a $(2 R+1)$-point rating scale; that is, for a 7-point scale, $R=3$. The scale midpoint must represent a neutral judgment. The data are arranged in a matrix $\mathbf{P}$, with rows belonging to assessors and columns belonging to the assessed. (Let $P_{i k}$ denote the rating given by assessor $i$ to group member $k$.)

The criteria for classification into sociometric status groups are applied to statistics that are derived from matrix $\mathbf{P}$. First, $R+1$ is subtracted from all values in $\mathbf{P}$; the resulting matrix $\mathrm{P}^{*}$ contains scores $-R, \ldots, 0, \ldots,+R$. By taking the absolute value of the elements of $P^{*}$ the matrix $I$ is created, which contains the impact scores $I_{i k}$. A matrix $\mathbf{S}$, which contains only non-negative scores $S_{i k}$ (sympathy ratings), is then created by substituting all negative scores by 0 in matrix $\mathbf{P}^{*}$. Similarly, a matrix $\mathbf{A}$ of antipathy ratings $A_{i k}$ is created by zeroing all positive scores and taking the absolute value of all remaining scores.

These manipulations of matrix $P$ are performed in the example of Table 1, which presents (1) fictitious scores of a person who is rated by nine group mates on a 7-point 
Table 1

Fictitious Ratings on a 7-Point Scale and Nominations Received by a Person From Nine Fellow Group Mernbers

\begin{tabular}{crrrrrrrrr}
\hline Person & $P$ & $P^{*}$ & $S$ & $A$ & $I$ & LM & LL & SP & SI \\
\hline A & 2 & -2 & 0 & 2 & 2 & 0 & 0 & 0 & 0 \\
B & 1 & -3 & 0 & 3 & 3 & 0 & 1 & -1 & 1 \\
C & 1 & -3 & 0 & 3 & 3 & 0 & 1 & -1 & 1 \\
D & 5 & 1 & 1 & 0 & 1 & 0 & 0 & 0 & 0 \\
E & 7 & 3 & 3 & 0 & 3 & 1 & 0 & 1 & 1 \\
F & 2 & -2 & 0 & 2 & 2 & 0 & 0 & 0 & 0 \\
G & 1 & -3 & 0 & 3 & 3 & 0 & 1 & -1 & 1 \\
H & 4 & 0 & 0 & 0 & 0 & 0 & 0 & 0 & 0 \\
I & 2 & -2 & 0 & 2 & 2 & 0 & 0 & 0 & 0 \\
Total & 25 & -11 & 4 & 15 & 19 & 1 & 3 & -2 & 4 \\
\hline
\end{tabular}

rating scale, and (2) fictitious concomitant least liked and most liked nominations given to the same person. The table also illustrates some common principles of the nomination method and our rating-scale method. It is seen that $P^{*}=S-A$ (analogous to the definition of social preference in the method of Coie et al., 1982) and $I=S+A$ (analogous to SI $=\mathrm{LM}+\mathrm{LL}$ in the method of Newcomb and Bukowski, 1983), both on the individual level of the assessor and on the aggregated level.

SSRAT, a probability method, can be regarded as a generalization of the method of Newcomb and Bukowski (1983). But whereas the latter method, under the null hypothesis of random attribution of nominations, is based on a fixed probability distribution (for each assessor) for the relevant statistics, SSRAT is predicated on the null hypothesis of conditional random attribution, a principle earlier applied by ten Brink (1985). This null hypothesis implies that each assessor randomly attributes ratings according to his/her particular probability distribution. Let us consider, for example, matrix $\mathbf{P}$ and assume for reasons of algebraic elegance only that $\mathbf{P}$ contains scores of the set $\{0, \ldots, 2 R\}$. Given the null hypothesis, the probability that an assessor $i$ will attribute a rating $r$ can be expressed as $p_{i}(r)$; hence,

$$
\begin{aligned}
& \operatorname{Prob}\left[P_{i k}=r\right]=p_{i}(r), \\
& \qquad \text { for } r=0,1,2, \ldots, 2 R .
\end{aligned}
$$

The parameters $p_{i}(r)$ are unknown and may be estimated from the data. Let $n_{i k}(r)$ be the variable counting whether or not assessor $i$ has given person $k$ a rating $r$; $p_{i}(r)$ is then estimated as follows:

$$
\hat{p}_{i}(r)=\sum_{k \neq i} \frac{n_{i k}(r)}{N-1} .
$$

$P_{+k}$, the rating total received by person $k$, can be taken as the sum of $N-1$ independent random variables $P_{i k}$; every $P_{i k}$ is drawn from the same value domain $\{0,1,2$, $\ldots, 2 R\}$, although with varying (over $i$ ) probability distribution. We work out the probability of realizing a certain value of $P_{+k}$ by means of mathematical induction.

For any assessed person $k$, the assessors are renumbered by index $j(j=1,2, \ldots, N-1)$, leaving the number of the assessed person out of consideration. Let $P_{k}(j)$ be the (sub)total score received by assessed subject $k$ from assessors $1,2, \ldots, j$. Then, for $j=1$ and $r=0,1,2, \ldots, 2 R$,

$$
\operatorname{Prob}\left[P_{k}(1)=r\right]=p_{1}(r) \text {. }
$$

And for $j=2,3, \ldots, N-1, r=0,1,2, \ldots, 2 R$, and $s=0$, $1,2, \ldots, \mathrm{j} * 2 R$,

$$
\operatorname{Prob}\left[P_{k}(j)=s\right]=\sum_{r} p_{j}(r) . \operatorname{Prob}\left[P_{k}(j-1)=s-r\right],
$$

where Prob $\left[P_{k}(j-1)=s-r\right]=0$, if $s-r<0$.

Moreover, for $j=N-1$ and $s=0,1,2, \ldots$, $(N-1)^{*} 2 R$,

$$
\operatorname{Prob}\left[P_{+k}=s\right]=\operatorname{Prob}\left[P_{k}(N-1)=s\right] .
$$

The expected value of $P_{+k}$ is now

$$
\mathscr{E} P_{+k}=\sum_{j} \sum_{r} r \cdot p_{j}(r) .
$$

In this notation, the successive order of assessors in matrix $\mathbf{P}$ plays a role. This means that $\operatorname{Prob}\left[P_{k}(j)=s\right]$ depends on the order selected. The end result $P_{+k}$, however, is irrespective of the order chosen, being the sum of the same $N-1$ independent random variables.

As an illustration, we fill out the formulae above as they apply to the probability that a person assessed by three others on a 3 -point rating scale $\{0,1,2\}$ receives a total score of 1 :

$$
\begin{aligned}
\operatorname{Prob}\left[P_{+k}=1\right]= & \operatorname{Prob}\left[P_{k}(3)=1\right] \\
= & p_{3}(0) \cdot \operatorname{Prob}\left[P_{k}(2)=1\right] \\
& +p_{3}(1) \cdot \operatorname{Prob}\left[P_{k}(2)=0\right] \\
= & {\left[p_{1}(1) \cdot p_{2}(0)+p_{1}(0) \cdot p_{2}(1)\right] \cdot p_{3}(0) } \\
& +p_{1}(0) \cdot p_{1}(0) \cdot p_{3}(1) .
\end{aligned}
$$

These expressions show that the probability distribution of $P_{+k}$ is captured entirely by $p_{i}(r)$. For these unknown parameters $\hat{p}_{i}(r)$ may be substituted.

The elaboration above was formulated with respect to matrix $\mathbf{P}$, but by analogy it also holds for matrix $\mathbf{I}$, if, in the formulae, $R$ is read instead of $2 R$. The same applies to matrices $\mathbf{S}$ and $\mathbf{A}$. In this way, probability distributions for the total scores $P_{+k}$ (total rating score), $I_{+k}$ (impact rating total), $S_{+k}$ (sympathy rating total), and $A_{+k}$ (antipathy rating total) of every assessed person are theoretically determined and estimated in practice.

To achieve sociometric status categorization, the actual values of these statistics need to be checked against criteria. The choice of these criteria is independent of the procedure followed above. The central role of the rating scores $P$ in our method and the analogy between the scores $P^{*}$ in our method and SP in the method of Coie et al. (1982), however, lead us to "translate" their criteria into probability terms ${ }^{1}$ :

$$
\text { 1. popular: } \begin{array}{r}
P_{+k} \text { significantly high, } \\
S_{+k}>\mathscr{E} S_{+k} \text { and } A_{+k}<\mathscr{E} A_{+k} ;
\end{array}
$$


2. rejected: $\quad P_{+k}$ significantly low, $S_{+k}<\mathscr{E} S_{+k}$ and $A_{+k}>\mathscr{E} A_{+k}$

3. neglected: $I_{+k}$ significantly low, $A_{+k}<\mathscr{E} A_{+k}$ and $S_{+k}<\mathscr{E} S_{+k}$;

4. controversial: $I_{+k}$ significantly high, $A_{+k}>\mathscr{E} A_{+k}$ and $S_{+k}>\mathscr{E} S_{+k}$

5. average: remaining group members.

SSRAT allows the researcher to make his/her own choice with respect to the significance level to be used in these criteria.

\section{Practical Implications}

For the method introduced in the previous section, a square matrix $\mathbf{P}$ is not essential. The underlying theory applies equally well if the group of assessors is a subset of the group of those assessed; in that case, the number of rows is less than the number of columns. The latter group may be a subset of the assessor group, in which case the number of rows exceeds the number of columns. In either case, matrix $\mathbf{P}$ may be conceived as rectangular, with an empty major diagonal in an enclosed square. All variants have in common that the set of assessors is different for each assessed group member. If the group of assessors is a subset of the group of assessed, even the number of assessors varies. These may be additional reasons to prefer a probability model such as SSRAT to a standard score method.

Optionally, SSRAT allows the application of the classification criteria set by Newcomb and Bukowski (1983). This is particularly useful when the researcher has collected "least liked" and "most liked" nominations and wishes to apply the probability method of Newcomb and Bukowski for nominations. Since SSRAT is a generalization of this method, the Newcomb and Bukowski procedure can be executed within the SSRAT framework, simply by using the following coding scheme: 1 = "least liked" nomination; 2 = no nomination; $3=$ "most liked" nomination.

The procedure that has been implemented in the SSRAT program can also be applied in many other situations where every member of a group is rated by other members of the group. Sociometric status is usually derived from the number of "least liked" and "most liked" nominations received. In sociometric research, different nomination criteria (such as "best friend," "least/most liked to play with") have a long history as well (Rogosch $\&$ Newcomb, 1989, p. 600). In fact, any aspect of social competence may be chosen as a criterion for judgment and presented to respondents with the request to nominate a number of group peers on the positive pole and on the negative pole, respectively. If respondents are asked to express an evaluative judgment of a group of objects (or persons) in terms of positive or negative nominations, these objects can always be assigned to the following categories: (1) objects that are nominated predominantly positively, (2) objects that are nominated predominantly negatively, (3) objects that are nominated frequently but diversely; and (4) objects that are nominated infrequently. Of course, for such an assignment, criteria for the categorization on the basis of the nominations have to be set in advance. Generally, a fifth category of objects that do not fit into any of these four groups will remain (Maassen et al., 1997).

The same can be achieved by asking respondents to rate their group peers on a scale. In a recent study of social competence of early adolescents, Maassen and Landsheer (1998) have taken the ability of adolescents to make acquaintances, as perceived by their peers, as a criterion of judgment. All pupils in the study were asked to rate whether they thought a particular classmate less competent than themselves, equally competent as themselves, or more competent than themselves in making acquaintances. Thus, a 3-point rating scale was involved, with a low attributed score corresponding to a relatively negative perception of the other's social competence. In the manner described above, application of SSRAT led to a classification into five categories of social competence, which may be labeled as follows: (1) high competence, (2) average competence, (3) low competence, (4) controversial, and (5) a group of youths who did not attract attention as particularly socially competent or incompetent, tentatively labeled as nondescript. The procedure leads to a classification into five categories, which should be labeled in accordance with the judgment criterion.

\section{Program Design}

Basically the program has a simple input-output structure. The program is written in Borland Pascal 7.0, using the TurboVision library, which facilitates the implementation of a modern user interface with a menu, dialog windows, and the use of a mouse. Input requirements should be flexibie, and therefore several options are included. The used rating scale can have $3,5,7$, or 9 points. The input of assessor-assessed matrices can have optionally either assessors in rows or assessors in columns, can contain missing data, and can be square or rectangular. Table 2 gives an example of the possible input.

Easy input also requires graceful handling of input errors. In addition, the program must be able to detect missing values and to handle them correctly. The input is checked for incorrect characters, depending on position.

Table 2

An Example of SSRAT Input Matrices for Two Groups 


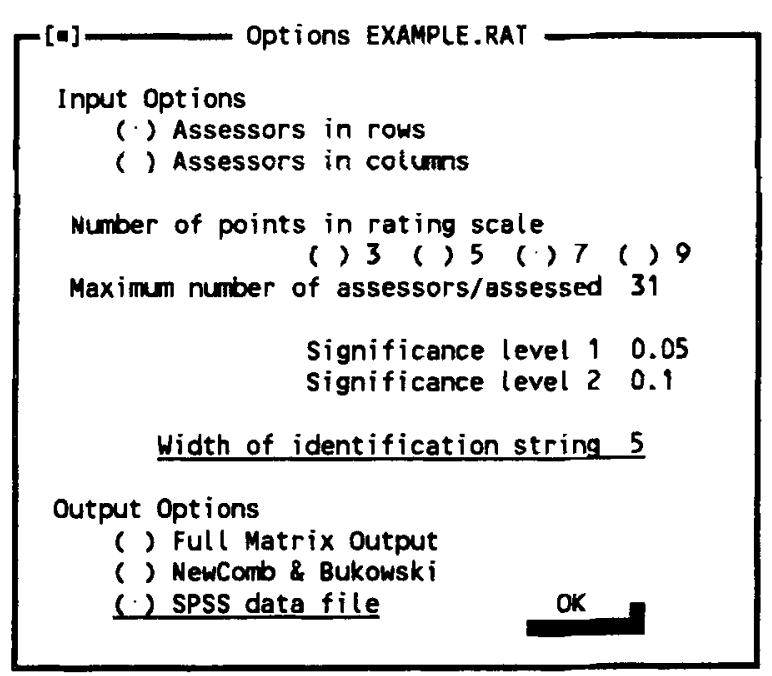

Figure 1. The Options window of SSRAT.

The first $n$ positions may contain an identification of optional length. The diagonal elements can be blank, or may contain a hyphen or a zero. All off-diagonal elements must contain a number within the range of the selected rating scale. All lines are checked for equal length. When an error is detected, the output contains a message indicating the error and its position.

Once the program starts, the user can enter the name of the input file with data matrices for analysis. Thereafter, a dialog window with all options is displayed (Figure 1).

The program calculates total scores for sympathy $(S)$, antipathy $(A)$, social preference $(P)$, and social impact $(I)$. It calculates for each assessed person probability distributions for $P, I, S$, and $A$, given the null hypothesis of conditional random attribution. From these distributions the probability of actual social preference and social impact scores and the expected scores for sympathy and antipathy are derived. According to the criteria set in advance, the probabilities of the observed $P$ and $I$ scores are checked against the chosen significance level, and the observed $S$ and $A$ scores are compared with the expected values. On the basis of the results, the sociometric status of every assessed person is determined.

The output is placed in a file, which is automatically loaded for inspection. Optionally, output can be made available in the form of an ASCII SPSS data file, which facilitates processing with a statistical package (Table 3).

All matrices $\mathbf{S}, \mathbf{A}, \mathbf{P}$, and $\mathbf{I}$ can be part of the output, as well as all results that are made available for further analysis: number of assessors, total sympathy score, total antipathy score, total social preference score, total social impact score, the probabilities referring to left-sided testing, the probabilities referring to right-sided testing, the expected values, and the sociometric status classification using the $\alpha$ that has been chosen in the Options dialog window. The user is enabled to study the output and to correct input errors as well as handling errors before ac- cepting the final output. The inclusion of a simple text editor facilitates the modification of input data and makes it possible to view the output.

\section{Intended Users}

Sociometric status determination is a customary procedure in educational or developmental psychological research. SSRAT may be very useful for students and investigators in these fields who wish to benefit from the advantages of rating scales. As a specific situation, we note that sociometric status in early childhood is often determined by means of a 3-point rating scale-that is, when children are asked to evaluate their peers with the help of three pictures (e.g., an unfriendly, a neutral, and a friendly face). (Asher et al., 1979; Hymel, 1983). SSRAT is an appropriate program for the analysis of the resulting data.

\section{Evaluation}

There has been some research to compare the results of SSRAT with those of the two nomination methods (Maassen, Akkermans, \& van der Linden, 1996). Comparison of the three methods in eight classroom groups, with a total number of pupils of 172 , showed that the Newcomb and Bukowski (1983) approach resulted in a considerably higher percentage of pupils being classified as average than that obtained with the rating-scale method implemented in SSRAT. A comparison of the social status categorizations resulting from application of the Newcomb and Bukowski method with SSRAT $(\alpha=.05)$ yielded a Cohen's $\kappa$ of only .27 . The similarity between the SSRAT classification $(\alpha=.05)$ and that of Coie et al. (1982) was also not very high: Cohen's $\kappa=.39$ (Maassen et al., 1996). Only 99 of the 172 pupils were classified the same. It is clear that the three methods yield distinctly different results.

Maassen et al. (1996) studied the validity of the three methods, using the average received rating as a criterion: The average received rating of a popular pupil should be relatively high and not lower than the neutral scale point, and the average rating of a rejected pupil should be relatively low and not higher than the neutral scale point. SSRAT with $\alpha$ set equal to .05 or lower proved to be a far

Table 3

An Example of the Output That SSRAT Can Produce

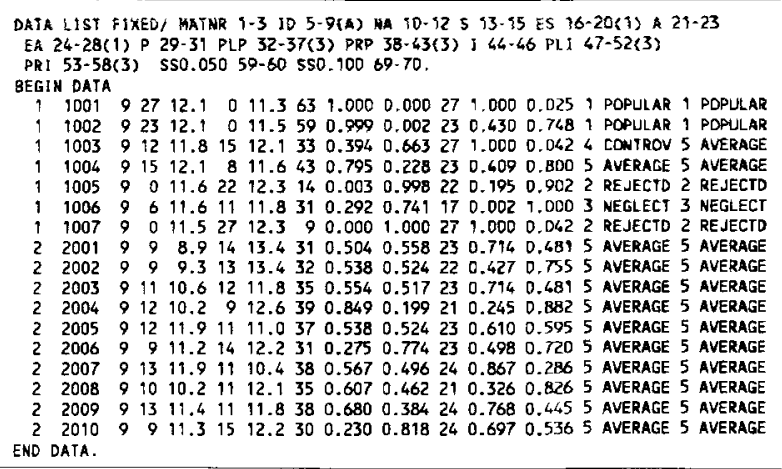


more effective way to determine populars, compared with the two nomination methods. In fact, the results of the study seem to indicate that populars should not be designated by the nomination methods. With regard to the rejected pupils, SSRAT with $\alpha=.025$ proved to be as effective as the nomination method of Coie et al. (1982), whereas the method of Newcomb and Bukowski (1983) turned out to be the more cautious procedure (i.e., yielding an appreciably lower number of rejected pupils with a lower mean average received rating).

\section{Documentation and Availability}

A 32-page manual is available (Maassen \& Landsheer, 1996), which explains the requirements for the input file, how the program can be used, the error messages that may occur, and the interpretation of the output, as well as the general handling of the program. Other relevant publications are ten Brink (1985), Maassen et al. (1996), and Maassen et al. (1997). The program and manual are available without charge. Send a DOS-formatted 1.44 MB 3.5-in. diskette with a self-addressed disk mailer to G. H. Maassen, Department Methodology and Statistics, Faculty of Social Sciences, Utrecht University, P.O. Box 80140 , 3508 TC Utrecht, the Netherlands (e-mail: g.maassen@ fss.uu.rl).

\section{REFERENCES}

ASHER, S. R., \& Hymel, S. (1981). Children's social competence in peer relations: Sociometric and behavioral assessment. In J. D. Wine \& M. D. Smye (Eds.), Social competence (pp. 125-157). New York: Guilford.

Asher, S. R., Singleton, L. C., Tinsley, B. R., \& Hymel, S. (1979). A reliable sociometric measure for preschool children. Developmental Psychology, 15, 443-444.

COIE, J. D., \& DODGE, K. A. (1983). Continuities and changes in children's social status: A five-year longitudinal study. Merril-Palmer Quarterly, 29, 261-282.

Coie, J. D., Dodge, K. A., \& Coppotelli, H. (1982). Dimensions and types of social status: A cross-age perspective. Developmental Psychology, 18, 557-570.

Hymel, S. (1983). Preschool children's peer relations: Issues in sociometric assessment. Merrill-Palmer Quarterly, 29, 237-260.
Maassen, G. H., Akkermans, W., \& van der Linden, J. L. (1996). Two-dimensional sociometric status determination with rating scales. Small Group Research, 27, 56-78

Maassen, G. H., Goossens, F. A., \& BoKhorst, J. (1998). Ratings as validation of sociometric status determined by nominations in longitudinal research. Social Behavior \& Personality, 26, 259-274.

MaAsSen, G. H., \& LandShEER, J. A. (1996). Manual for SSRAT 2.0: A program for two-dimensional sociometric status determination with rating scales. Utrecht: FSW/ISOR Publications.

MAassen, G. H., \& LANDSheer, J. A. (1998). The relationship between peer-perceived social competence and school success for low educated young adolescents. Manuscript submitted for publication.

MaAssen, G. H., van der Linden, J. L., \& AkKermans, W. (1997). Nominations, ratings and the dimensions of sociometric status. International Journal of Behavioral Development, 21, 179-199.

NewCOMB, A. F., \& BUKowski, W. M. (1983). Social impact and social preference as determinants of children's peer group status. Developmental Psychology, 19, 856-867.

Newcomb, A. F, Bukowski, W. M., \& Pattee, L. (1993). A metaanalytic review of popular, rejected, neglected, controversial, and average sociometric status. Psychological Bulletin, 113, 99-128.

Rogosch, F. A., \& Newcomb, A. F. (1989). Children's perceptions of peer reputations and their social reputations among peers. Child Development, 60, 597-610.

TEN BRINK, P. W. M. (1985). De gegeneraliseerde binomiale verdeling als alternatief voor de sociometrische status berekening volgens het probabiliteitsmodel [The generalized binomial distribution as an alternative for sociometric status determination according to the probability model]. Unpublished master's thesis, Psychologisch Laboratorium K.U.N., Nijmegen.

TERRY, R., \& CoIE, J. D. (1991). A comparison of methods for defining sociometric status among children. Developmental Psychology, 27, 867-880.

\section{NOTE}

1. 'Translation' of the criteria by Coie et al. (1982) according to the following rules:

$z$ score $<-1$ becomes: raw score significantly low;

$z$ score $>+1$ becomes: raw score significantly high;

$z$ score $<0$ becomes: raw score less than the expected value;

$z$ score $>0$ becomes: raw score greater than the expected value.

(Manuscript received April 16, 1997; revision accepted for publication September 4, 1997.) 